\title{
A geometric morphometric study into the sexual dimorphism of the human scapula
}

Y. Scholtz, M. Steyn*, E. Pretorius

Forensic Anthropology Research Centre, Department of Anatomy, University of Pretoria,

P.O. Box 2034, Pretoria, 0001, South Africa

Abbreviated title: Sexual dimorphism of the scapula

Corresponding author:

Prof. Dr. Maryna Steyn,

Tel: 27-12-4203256

Fax: 27-12-3192240

E-mail: maryna.steyn@up.ac.za

No. of pages (text): 18

No. of tables: 2

No. of figures: 11 


\begin{abstract}
Sex determination is vital when attempting to establish identity from skeletal remains. Two methodological approaches to sex determination exist: morphological and metrical. The aim of this research paper was to use geometric morphometrics to study the shape of the scapula and its effect on the sexual dimorphism the scapula. The sample comprised of 45 adult black male and 45 adult black female scapulae of known individuals. The scapulae were photographed and 21 homologous landmarks were plotted to use for geometric morphometric analysis with the 'tps' series of programs, as well as the IMP package. Consensus thin-plate splines and vector plots for males and females were compared. The CVA and TwoGroup analyses indicated that significant differences exist between males and females. The lateral and medial borders of females are straighter while the supraspinous fossa is more convexly curved than that of males. More than $91 \%$ of the females and $95 \%$ of the males were correctly assigned. Hotelling's $\mathrm{T}^{2}$-test yielded a significant $\mathrm{p}$-value of 0.00039 . In addition, 100 equidistant landmarks representing the curve only were also assigned. These, however, yielded considerably poorer results. It is concluded that it is better to use homologous landmarks rather than curve data only, as it is most probable the shape of the outline relative to the fixed homologous points on the scapula that provides the separation.
\end{abstract}




\section{Introduction}

The correct determination of sex is vital when attempting to establish identity from skeletal remains, since this can cut the number of possible matches in half. Traditionally the skull, pelvis and long bones are used for identification purposes and metric and morphological methods are employed as identification tools. However, other bones in the body may also provide useful information about sex. Therefore, in this study, the shape of the scapula was studied in order to gain information on its sexual dimorphism.

Studies that have been performed previously on the scapula, specifically to investigate its sexual dimorphism, have yielded mixed results. Research into the sexual dimorphism of the scapula include that of Bainbridge and Genovés (1956), Churchill et al. (2001), Frutos (2002), Getz et al. (1996), Graves (1921) and Hrdlička (1942a, 1942b). There are, however, currently no existing methods for determining sex using the shape of the scapula. If such differences can be found, it could improve the results obtained when skeletal material is analyzed for identification of the individual.

One problem that is frequently encountered by researchers when analyzing biological data is the assessment of similarity between groups of objects. Methods that produce qualitative results do not provide statistical tests of group differences and researchers may draw different conclusions from the same results (Coward and McConathy, 1996). Morphological analysis is dependent on the experience of the observer and can be influenced by inter- and intraobserver errors, as well as problems with standardization and statistical analysis (Coward and McConathy, 1996; Frutos, 2002; Loth and İşcan, 2000). Two methodological approaches to sex determination exist: morphological and metrical (e.g., Coward and McConathy, 1996; Frutos, 2002; Loth and İşcan, 2000). Morphological techniques are qualitative and focus on shape. These techniques usually involve the pelvis and cranium. Metrical analysis is quantitative, focusing on bone dimensions. It is usually employed when the bones are in a fragmentary state, or when analyzing long bones that do not show many morphological differences (e.g., Frutos, 2002; Loth and İşcan, 2000). A combination of measurements can also be selected to maximize sex diagnosis by using discriminant function statistics. A major problem with this technique is that metric standards are population specific.

Another technique, namely geometric morphometrics, has been used successfully to determine and quantify morphological similarities and differences in biological material (e.g., Bastir and Rosas, 2004; Bookstein et al., 2003; Hennessy and Stringer, 2002; O'Higgins et al., 2001; Oettlé et al., 2005, 2009; Pretorius and Scholtz, 2001; Pretorius et al., 2006; Steyn et 
al., 2004; Strand Viðarsdóttir et al., 2002). This technique uses x/y co-ordinates or landmarks to quantify shape, and is particularly valuable when studying shape that forms bulges and curves that are difficultly quantifiable using traditional metric measurements (Bastir and Rosas, 2004; Bookstein, 1990; Rohlf, 2003).

Studies using geometric morphometrics to investigate sexual dimorphism include that of Hennessy et al. (2002), Oettlé et al. (2005, 2009), Pretorius et al. (2006) and Steyn et al. (2004). Sliding semi-landmark geometric morphometric methods have also been employed in a number of studies (De Groote et al., 2010; Gonzalez et al., 2009; Perez et al., 2006). The sliding semi-landmark method is used when the structure being studied has too few or no identifiable landmarks.

Lele and McCulloch (2002) defined the form of an object as the characteristic that remains invariant under a group of transformations including rotation, translation and reflection. They examined methods for the statistical analysis of form from the invariance perspective. Various methods for the comparison of forms in single- and two-sample cases were examined and it was found that methods based on distances between landmarks are more reliable than superimposition and deformation methods and that these methods should be analyzed carefully with regard to invariance and identifiability.

Although there have been previous studies into the sexual dimorphism of the scapula, none of these used geometric morphometrics. Therefore, the aim of this research was to use geometric morphometrics to investigate the shape of the scapula in order to determine differences or similarities between male and female scapular shape. Although geometric morphometric software supports analysis in three dimensions, this study focused on a two dimensional analysis mostly assessing the shape of the scapular body, excluding the spine and acromion.

\section{Materials and methods}

The scapulae in this study originated from the Pretoria Bone Collection at the Department of Anatomy, University of Pretoria (L'Abbé et al., 2005). The left scapulae of 45 adult black males and 45 adult black females were used. The ages ranged from 20-96 for males and 2879 for females. Only scapulae of known individuals were used and damaged scapulae or scapulae showing pathology were excluded, as this could have influenced the shapes of the scapulae which, in turn, could have influenced the outcome of this study. 
In order to standardize the position of the scapulae, the bones were placed on graph paper on an osteometric board with the dorsal side facing upwards. The lateral border was placed against the vertical surface of the osteometric board, so that the glenoid fossa and inferior angle rested against the vertical surface.

Photographs were taken of the dorsal surface of the scapulae with an Olympus D-395 digital camera which was mounted in a fixed position. The camera was positioned at a height of $470 \mathrm{~mm}$ directly above the scapulae. The viewfinder of the camera was focused on a marked spot on the graph paper to ensure that the photographs would be taken in the same position each time.

\section{Traditional landmark data collection}

The captured images were transferred to a computer and 21 homologous landmarks were assigned to each scapula to use for geometric morphometric analysis (Fig. 1). The landmarks were selected so that they would be easily identifiable, and would reflect the shape of the body of the scapula.

The landmarks were positioned by eye, and are as follows:

Landmark 1: On the glenoid fossa, at the point where it touches the vertical surface of the osteometric board.

Landmark 2: At the position where the lateral border touches the vertical surface of the osteometric board near the inferior angle of the scapula.

Landmarks 3 and 4: At equal distances between landmarks 1 and 2 on the lateral border. Landmark 5: On the most inferior part of the inferior angle, visualized using the graph paper as guide.

Landmark 6: At an equal distance between landmarks 2 and 5 on the lateral border.

Landmark 7: At the position where the spinous process ends on the medial border of the scapula. It was assigned by following the scapular spine through to where it would reach the medial border of the scapula, taking into account the way in which it sometimes splits to form a triangular area.

Landmarks 8 and 9: At equal distances between landmarks 5 and 7 on the medial border. Landmark 10: At the point where the superior border of the scapula reaches the spine. It was assigned by following the superior border of the scapula laterally towards the scapular spine, at the point where the superior border "disappears" behind the scapular spine.

Landmark 11: Along the superior border at an equal distance between landmarks 7 and 10. 
Landmarks 12 and 13: At equal distances between landmarks 10 and 11.

Landmarks 14 and 15: Similarly situated between landmarks 7 and 11.

Landmark 16: Situated at an equal distance between landmarks 10 and 12.

Landmark 17: Similarly situated between landmarks 12 and 13 (This is not necessarily the highest point on the scapula, although it might appear that way on some scapulae - this depends on the shape of the supraspinous fossa).

Landmark 18: Situated at an equal distance between landmarks 11 and 13.

Landmark 19: Similarly situated between landmarks 11 and 14.

Landmark 20: Situated equidistantly between landmarks 14 and 15.

Landmark 21: Similarly situated between landmarks 7 and 15.

It should be noted that landmarks 11-21 are not necessarily exactly equidistant, as it is almost impossible to assign equidistant landmarks on a curve. This fact is not really relevant, as these landmarks are only present to ensure that a landmark is present along every few millimetres of the supraspinous border.

The tps series of programs (versions by F. James Rohlf indicated where applicable) was used to analyze the shapes. The homologous landmarks described before, were digitized using the tpsDig program (F. James Rohlf, Version 1.31). Because shape differences between males and females were studied, the average, or consensus configuration of landmarks for each of these two groups, was computed using tpsSuper (F. James Rohlf, Version 1.03). From this it was possible to visually assess whether any differences exist between the males and females.

The consensus configurations of the two groups were compared with each other in order to determine which landmarks were responsible for the variation. Thin-plate splines were calculated using tpsSplin (F. James Rohlf, Version 1.14). This program determined another consensus configuration representing the average shape of the two groups (or average of the whole population) and was represented by a perfect perpendicular grid. Deformation of the grids of the consensus configurations of the two groups allowed the researchers to determine the locations of the variation. The consensus thin-plate splines were also viewed as vector plots to determine which landmarks were responsible for the greatest amount of variation.

Sheets' Integrated Morphometric Package (IMP) TwoGroup-program and Canonical Variates Analysis-program (CVAGen6) were used for calculation of significance levels between male and female shape (Sheets, 2001). CVAGen6 was also used to perform a discriminant function analysis and to generate a plot indicating the similarities or differences in the clusters of landmarks of the different groups. This plot can be given in the shape of the 
structure that is being investigated, or the program can generate a CVA-plot that simply shows whether any overlap is present in the clusters of landmarks by grouping all the individuals in the different groups on separate sides of their mean shape. The amount of overlap can be used to determine the extent of any differences.

CVAGen6 can also calculate a p-value to show if the differences between the two groups are statistically significant. A CVA assesses the ability to assign specimens in a dataset to groups (e.g. male or female), rather than asking if the two groups have a different shape. The program computes partial warp scores to a common reference and determines how many CVA axes there are in the data at a $\mathrm{p}=0.05$ level of significance. It then computes the canonical variate scores of all the specimens entered (Sheets, 2001).

TwoGroup6 forms part of the IMP suite for the analysis of 2D landmark based geometric morphometric data. It was used to determine how the males and females would group. TwoGroup offers a variety of statistical tests (including Hotelling's $\mathrm{T}^{2}$-test for significant differences in shape between two groups). It also calculates the Full and Partial Procrustes Distances between the means of two groups, as well as a bootstrap estimate of the variance in this distance (Sheets, 2001).

TwoGroup uses Hotelling's $\mathrm{T}^{2}$-test and Goodall's F-test to calculate p-values in order to determine statistical significance. Goodall's F-test tests for inter-group shape difference between the population groups. It tests for overall shape difference between groups taking sample variance into account. It compares the Procrustes distance between the means of two samples to the amount of variation found in the samples. Generalized least-squares Procrustes analysis is used to compute the average shape for each sample (Hennessy and Stringer 2002; Loy et al., 1999; Rohlf, 2000).

Hotelling's $\mathrm{T}^{2}$-test compares an observed mean vector to a parametric mean; or the difference between two mean vectors to a parametric difference (Slice et al., 1998, 2009).

The inter-group shape differences between the males and females were tested by means of the TwoGroup-program. Hotelling's $\mathrm{T}^{2}$-test and Goodall's F-test were used to test for statistical significance and the results were visualized by means of a TwoGroup BC Superimposition.

The TwoGroup results were visualized by BC Superimpositions. BC is the abbreviation of “Bookstein Coordinates, which are also known as Bookstein's shape coordinates or Two point shape coordinates (originally from Francis Galton, but rediscovered by Bookstein). Bookstein Coordinates are described as a system of shape coordinates consisting (for twodimensional data) of the coordinates of landmarks $3,4 \ldots 21$ after the forms are rescaled and 
repositioned so that landmarks 1 and 2 are fixed at $(0,0)$ and $(1,0)$ respectively, in a Cartesian coordinate system (Sheets, 2001; Slice et al., 1998, 2009).

On the BC Superimposition plot the clusters of landmarks of the two groups are superimposed onto one another, and the differences and/or similarities can be seen in the separation of their means relative to how tightly they are clustered together.

To test for intra- and inter-observer repeatability, 15 male and 15 female scapulae were randomly selected and re-assigned the chosen landmarks by the same and a different observer. The "new" landmark data was statistically compared to the "old" dataset using Hotelling's T² test and Goodall's F-test of the TwoGroup program.

\section{Curve landmark data collection}

A second method was also employed, where the curve function in tpsDig was used. The curve was plotted using 100 landmarks and the starting landmark was landmark 1 (as described above), while the curve ended with landmark 10. The curve dataset was converted to landmarks in the tps Utility program (tpsUtil version 1.38). The purpose of this program is to provide various file conversions and utility functions in order to manipulate the tps files that are used in the rest of tps series of morphometric programs. In tpsRelw, the converted tps landmark file was loaded and a consensus shape generated for both the males and the females. The consensus for the males and females were viewed in tpsSplin. Using the TwoGroupprogram from the IMP series of programs, a p-value was obtained.

\section{Results}

\section{Traditional landmark data results}

To test for intra-observer repeatability of placement of landmarks, 15 male and 15 female scapulae were randomly selected and re-assigned the chosen landmarks by the same observer. The "new" landmark data were statistically compared to the "old" dataset using Hotelling's $\mathrm{T}^{2}$-test and Goodall's F-test of the TwoGroup program. Hotelling's $\mathrm{T}^{2}$-test yielded a p-value of 0.08624 , while Goodall's F-test yielded a p-value of 0.77358 , indicating that there is no statistically significant difference between the two sets of landmarks and, thus, that intraobserver repeatability is possible. 
To test for inter-observer repeatability the 30 scapulae mentioned above were once again selected and the landmarks were reassigned by a different observer. The "new" landmark data (as assigned by the independent observer) were once again statistically compared to the original dataset. Statistically significant differences were found between the two datasets (Hotelling's $\mathrm{T}^{2}$-test: $\mathrm{p}=3.1496 \times 10^{-8}$; Goodall's F-test: $\mathrm{p}=3.052 \times 10^{-13}$ ). The landmarks where the biggest difference between original and independent observer were seen, were landmarks 3, 4, 7 and 9. These landmarks are situated on the lateral (3 and 4) and medial borders (7 and 9). These discrepancies will be further addressed in the Discussion.

Figures $2 \mathrm{a}$ and $2 \mathrm{~b}$ represent the thin-plate splines for female and male scapulae respectively. The grid is indicated in solid lines to give a clearer indication of the deformation of the female and male grids respectively and also to indicate that, while the female shape seems to be perfectly perpendicular, there is in fact deformation of the grid. When these images are compared, it is clear that there are differences between the two sexes, especially along the superior border of the scapula, but these differences can be seen more clearly in the vector plot for male and female scapular shape (Fig. 3). From this vector plot it can be seen that the female shape is narrower, while the male shape is broader. The male lateral border is slightly more curved than that of the female, while the inferior angle of the female seems less sharp than that of the male. The female medial border is straighter than in males, while the superior border is somewhat more convex than that of the males.

Hotelling's $\mathrm{T}^{2}$-test was performed using the TwoGroup program and a $\mathrm{p}$-value of 0.00039 was obtained. This value indicates that there is a statistically significant difference between the shapes of male and female scapulae.

Figure 4 is a representation of the mean CVA plot for females and males, with the females indicated by circles and the males by crosses. The larger circle and cross in the centre of the CVA plot represent the mean shape for the females and males respectively. On this CVA plot the separation between males and females can clearly be seen with almost no overlap present.

Based on the above-mentioned differences, a CVA analysis was carried out to test the accuracy with which a scapula can be categorized as male or female. Table 1 represents the accuracies obtained from this analysis. Forty-one of the female scapulae were correctly assigned as female (91.1\% accuracy), while 43 of the male scapulae were correctly assigned as male (95.6\% accuracy). The p-value obtained by the CVA analysis was 0.00015 , which indicates that the sexual dimorphism of the scapula is statistically significant.

In order to understand the observed differences better, the three borders of the male and female scapula were studied separately. The lateral and medial borders were not found to 
show statistically significant differences between the two sexes, but the superior border of the supraspinous fossae did.

When the consensus thin-plate splines for the supraspinous fossae of females and males (landmarks 7, 10-21), as well as the vector plot for the two groups are compared (Figs. 5a and b and Fig. 6), the separation between the female and male supraspinous fossa borders becomes clear. From the vector plot it can be seen that males differ from females at all the landmarks, especially landmarks 7, 10, 12,15-17, 20 and 21. The superior border of the male supraspinous fossa seems to run along a downward slope towards the superior angle, while that of the female has a convex shape. The medial border of the male is more curved towards the scapular spine, while that of females seems to be straighter (Goodall's F-test: $p=0.038$; Hotelling's $\mathrm{T}^{2}$-test: $\mathrm{p}=0.063$ ).

Figure 7 is the mean of the CVA plot for the supraspinous fossa of females and males, with females once again indicated by circles and males by crosses. Some separation can be seen between the two sexes, but a larger degree of overlap is now present.

\section{Curve landmark data results}

Figure $8 \mathrm{a}$ and $\mathrm{b}$ shows the consensus thin-plate spline for the curve data of the female and male scapula. These splines look similar to the landmark data splines (Figs. 2a and b). However, the Goodall p-value was 0.922, which suggests that the males and females are not significantly different using this method. The CVA analysis yielded classification accuracies of $64.44 \%$ in both males and females (Table 2).

In order to try to explain the discrepancy between the two methods employed, the two consensus thin-plate splines in Figure 8 (the curve data for females and males) were viewed as a vector plot (Fig. 9). This vector plot is similar to the vector plot that was calculated for male and female scapulae using the traditional landmark method (Fig. 3). From this image it can be seen that there are differences between the shapes of the two sexes, and that it is more clearly visible once the curves are aligned relative to the two fixed landmarks. The female scapular shape seems broader than that of males, while the male lateral and medial borders are more curved than those of the females. The differences originally seen in the superior border of the supraspinous fossa are also present, with the superior border of the females displaying a more pronounced curve than that of the males (which shows a downward-sloping superior border).

\section{Discussion}


Statistically significant differences were found between the shapes of male and female scapulae using homologous landmarks. Figures 10 and 11 are examples of typical female and male scapulae (Bass, 1995; El-Najar and McWilliams, 1978; Prescher, 2000; Scheuer and Black, 2000). When looking at the complete female scapula, it is much narrower than that of a male. The lateral border is straighter in the female and more curved in the male. The inferior angle is generally sharper in males than in females and the medial border of the male is more curved than that of the female - broader towards the scapular spine, and narrower towards the inferior angle. The superior border of the supraspinous fossa in females may initially have a somewhat concave course, but then shows a convex curve at the superior angle, while in males it runs with a downward slope towards the superior angle. The medial border of the supraspinous fossa is straighter in females, while in males it curves toward the scapular spine. However, much variation and overlap exists.

As described in the results, one of the problems in this study was that it is difficult to assign homologous landmarks to the scapula. There are few definite homologous landmarks along the borders of the scapula and many of the landmarks are to a large extent dependent upon the observer (except for landmarks 1, 2, 5 and to some extent 7 and 10 which remain constant). This may have influenced the results, although there is currently no way to bypass these problems. When testing intra-observer repeatability, no significant differences were found between the "new" and "old" datasets. Statistically significant differences were, however, found between the original landmarks and those assigned by an independent observer. The biggest differences were found at landmarks 3, 4, 7 and 9. These landmarks were situated on the lateral (landmarks 3 and 4) and medial borders (landmarks 7 and 9). One possible reason for the discrepancies in the assignment of landmarks 3, 4 and 9 is that these three landmarks were not assigned to fixed points on the scapula, but were placed midway between fixed landmarks. The assignment of landmark 7 could also present problems as there is no real fixed point where the spinous process ends on the medial border and the landmark had to be assigned by following the spine through to where it reaches the medial border and in some cases forms a triangular area on the medial border. In this respect curve landmark data seems to have the advantage, as problems with repeatability are ruled out.

In order to understand the observed differences between male and female scapulae better, the three borders (lateral, medial and superior) were studied separately. The same differences were seen when the three borders were studied separately as when the complete scapula was 
studied, with the superior border showing the most variation between the two sexes. However, the combination of the three borders yielded much better results than when they were studied separately, indicating that all areas contribute towards the dimorphism.

One of the drawbacks to studying the three borders separately was that the three borders on their own did not yield good results when using the Integrated Morphometrics Package (IMP). Neither the TwoGroup program's statistical analysis nor the CVA analysis could be used on the medial border as there were not enough landmarks. Although the two programs were used on the lateral border and supraspinous fossa, the results obtained were not significant. The lateral border yielded non-significant p-values. A CVA plot for the mean shapes of the female and male lateral borders showed almost complete overlap.

The TwoGroup program was used with some success on the supraspinous fossa, with significant levels of differences found. A CVA plot for the mean shapes of the female and male supraspinous fossae, however, showed a fairly large degree of overlap. This indicates that the superior border of the scapula is a more accurate indicator of sexual dimorphism than the other borders.

When the medial border of the scapula (pooled sexes) was compared with results obtained by Graves (1921), some similarities were seen. Graves found that the convex shape of the medial border was most common (61-54.3\% of individuals), with a straight medial border the second most frequent (26-27\%). The concave medial border was the least frequent (13$18.7 \%$ of individuals). In the current study similar results were seen in the pooled sample group, with $53.3 \%$ of the sample showing a convex medial border, $34.4 \%$ showing the straight medial border and $12.2 \%$ a concave medial border.

Five scapular body shapes were identified by Hrdlička (1942): triangular or wedge-shaped with a straight medial border (type 1), concave or bi-concave with a concave medial border (type 3 ) and convex with a convex medial border (type 6). The body of the scapula was also found to sometimes be quadrilateral with the axillary border augmented by a distinct inferior border (type 4). The pentagonal shape occurs when the type 4 scapula is augmented by a distinct angle in the vertebral border at the terminal point of the spine to form two wellmarked borders (type 5). Hrdlička named types 1, 3 and 6 the main scapular shapes and noted that any of these three types may be accompanied by one or both of the additional fourth (antero-inferior) and fifth (postero-superior or epispinous) borders. He found that sex differences did exist between the different scapular shapes, with type 1 being more frequent in females and types 3 and 6 more common in males. These differences were, however, not significant. 
The geometric morphometric analysis in the current study also indicated that the medial border of the scapula was straighter in females and more curved in males (broader towards the scapular spine and narrower towards the inferior angle). These results are supported by Bainbridge and Genovés (1956), who assessed the scapulae from a collection of $17^{\text {th }}$ and $18^{\text {th }}$ century skeletons from St. Bride church in London. They recognized the three main forms of the infraspinous portion of the medial border as convex, straight and concave and found that the male scapula was convex in 37 of the 45 male scapulae, while the medial border of females was straight in seven of the 13 female samples. Krobot et al. (2009) investigated the shape of the scapula and its relation to the performance of the muscles of the shoulder. They also identified the standard male shape as having a convex medial border and the standard female shape as having a straight medial border.

In this study, the superior border of the scapula was found to be convexly curved in females, while in males it runs with a downward slope towards the superior angle. These results are supported by Hrdlička (1942a), who also found some sexual dimorphism in the superior border of the scapula. He identified six types of superior border: horizontal or slightly rising (type 1); moderately rising or oblique (type 2); markedly oblique or steep (type 3); angular or deep saddle-shaped (type 4); markedly concave or semi-lunar (type 5) and markedly concavo-convex or wavy (type 6). Hrdlička found that the type 1 and 2 superior border was more frequent in females while types 3, 4 and 5 were seen more frequently in males. Type 6 was seen only rarely in males and was completely absent in females. In this study the superior border of the scapula was found to be the most variable of all the borders.

The percentage accuracies as indicated by the CVA analysis (95\% males, $91 \%$ females) compare very favourably with previous studies using geometric morphometric techniques to determine sexual dimorphism. These include studies of the greater sciatic notch $(87.1 \%$ females, $93.1 \%$ males), mandibular ramus flexure (67.8\% females; $69.9 \%$ males) and orbital shape (80.0\% females, 73.3\% males) (Oettlé et al., 2005, 2009; Pretorius et al., 2006; Steyn et al., 2004).

Compared to the above, the results obtained from the current study on the sexual dimorphism of the scapula are very accurate, indicating that the shape of the scapula can be used as an indicator of sex. These results also indicate that geometric morphometric methods can be used to accurately determine sex from scapular shape. These methods can, however, only be used on the complete scapula, as the results obtained from the three separate borders, except for the superior border, were not statistically significant. 
Geometric morphometric techniques could be used, for example, if a complete scapula of unknown origin was found. The scapula can be photographed in the standardized position described above and the same landmarks assigned. The unknown scapula can then be pooled with a selection of known scapulae to determine into which group a CVA analysis classifies it. However, more research is needed to see how accurately it can be assigned as male or female when traditional morphological assessments are made.

The differences between the male and female scapulae might partially be the result of males being more active and doing more physical labour, leading to better developed muscles. The influence of surrounding muscles on scapular shape is supported by Kuhns (1945) and Wolffson (1950), both of whom stated that the attaching muscles of the vertebral border played a role in determining its shape. Kuhns (1945) found that the concave shape of the vertebral border occurred due to poor muscle development. He also thought of the concave vertebral border shape as a juvenile variant of the mature form and indicated that it could be caused by the wide separation of the insertions of the rhomboid muscles. The convex medial border was seen to be caused by maximum development of the powerful muscles that surround the shoulder girdle. Although he could not find any variation in the muscular attachment to the scapula in his sample, Kuhns believed that the variations in the vertebral border were the result of changes in muscle function.

Hrdlička (1942b) stated that the cause of the sexual dimorphism in scapular shape seemed to be ontogenetic due to the fact that he saw shape differences between males and females in each of the population groups that he studied, as well as differences in the scapular shape between females from different population groups, while the males in the different population groups remained similar in shape. He also stated that the shape of the superior border was partially hereditary and partially acquired, with the hereditary shapes being type 1, 2, 4 and 5 and types 3 and 6 being the acquired shapes.

This, however, does not fully explain the differences and further research into this topic is needed. It should also be verified whether the same patterns can be seen in other populations.

The curve data method that was used in this study was similar to the methods used by Perez et al. (2006), however, in this study sliding semi-landmarks were not used, as these methods could not be analyzed using the IMP package.

When looking at the consensus thin-plate splines for the curve data, some differences can be seen between males and females. These differences, however, are not as prominent as those seen in the consensus thin-plate spline for the traditional landmark data and were not statistically significant. When the vector plot for female and male curve data is viewed, 
however (Fig. 9), the difference between the two groups can be seen more clearly. These results suggest that while curve data alone could be used, the results would be better if combined with traditional landmark data as a reference. It seems as if it is the shape of the curves, relative to specific homologous landmarks, that contributes towards the differences between the sexes. Further studies might be done where traditional landmarks (e.g. the landmarks at definite points on the scapula, for instance landmarks 1, 2, 5, 7 and 10) are combined with curve data. More research is needed using different anatomical structures.

\section{Acknowledgements}

This work was supported by grants from the South African National Research Foundation (NRF), as well as the Research Committee of the University of Pretoria (NAVKOM). We would also like to thank the Department of Anatomy, University of Pretoria for use of the Pretoria Bone Collection. We are also grateful to V. Wanek-Vance for help with the testing of inter-observer repeatability of the landmarks and to Profs. Rohlf and Sheets for their help in the implementation of the various programs. The authors would also like to thank the two anonymous reviewers for valuable contributions to this paper.

\section{References}

Bainbridge, D., Genovés, S., 1956. A study of sex differences in the scapula. J. Royal Anthropol. Institute 86, 109-134.

Bass, W.M., 1995. Human Osteology: A Laboratory and Field Manual. Missouri Archaeological Society, Columbia.

Bastir, M., Rosas, A., 2004. Facial heights: Evolutionary relevance of postnatal ontogeny for facial orientation and skull morphology in humans and chimpanzees. J. Hum. Evol. 47, $359-381$.

Bookstein, F.L., 1990. Introduction to Methods for Landmark Data. In: Rohlf, F.J., Bookstein, F.L. (Eds.) Proceedings of the Michigan Morphometrics Workshop; 1988 May 16-28; Ann Arbor, Michigan. University of Michigan Museum of Zoology, Ann Arbor, pp. 215-225.

Bookstein, F.L., Gunz, P., Mitterœcker, P., Prossinger, H., Schæfer, K., Seidler, H., 2003. Cranial integration in Homo: singular warps analysis of the midsagittal plane in ontogeny and evolution. J. Hum. Evol. 44, 167-187. 
Churchill, R.S., Brems, J.J., Kotschi, H., 2001. Glenoid size, inclination, and version: An anatomic study. J. Shoulder Elbow Surg. 10, 327-332.

Coward, W.M., McConathy, D., 1996. A Monte Carlo Study of Inferential Properties of Three Methods of Shape Comparison. Am. J. Phys. Anthropol. 99, 369-377.

De Groote I, Lockwood CA, Aiello LC. 2010. Technical note: A new method for measuring long bone curvature using 3D landmarks and semi landmarks. Am. J. Phys. Anthropol. $141,658-664$.

El-Najar, M.Y., McWilliams, K.R., 1978. Forensic Anthropology: The Structure, Morphology, and Variation of Human Bone and Dentition. Charles C Thomas, Springfield. Frutos, L.R., 2002. Determination of Sex from the Clavicle and Scapula in a Guatemalan Contemporary Rural Indigenous Population. Am. J. Forensic Med. Pathol. 23, 284-288.

Getz, J.D., Recht, M.P., Piraino, D.N., Schils, J.P., Latimer, B.M., Jellema, L.M., et al., 1996. Acromial Morphology: Relation to Sex, Age, Symmetry, and Subacromial Enthesophytes. Radiology 199, 737-742.

Gonzalez PN, Bernal V, Perez SI. 2009. Geometric morphometric approach to sex determination of human pelvis. Forensic Sci. Int. 189, 68-74.

Graves, W.W., 1921. The types of scapula. Am. J. Phys. Anthropol. 2, 111-128.

Hennessy, R.J., Kinsella, A., Waddington, J.L., 2002. 3D Laser Surface Scanning and Geometric Morphometric Analysis of Craniofacial Shape as an Index of Cerebrocraniofacial Morphogenesis: Initial Application to Sexual Dimorphism. Biol. Psychiatry 51, 507-514.

Hennessy, R.J., Stringer, C.B., 2002. Geometric morphometric study of the regional variation of modern human craniofacial form. Am. J. Phys. Anthropol. 117, 37-48.

Hrdlička, A., 1942a. The scapula, visual observations. Am. J. Phys. Anthropol. 29, 73-94.

Hrdlička, A., 1942b. The adult scapula, additional observations and measurements. Am. J. Phys. Anthropol. 29, 363-415.

Krobot, A., Janura, M., Elfmark, M., 2009. Functional categorization of the individual morphology of the scapula. Med. Biol. Eng. Comput. 47, 497-506.

Kuhns, J.G., 1945. Variations in the vertebral border of the scapula: their relation to muscular function. Physiotherapy Review 25, 207-210.

L'Abbé, E.N., Loots, M., Meiring, J.H., 2005. The Pretoria Bone Collection: A modern South African skeletal sample. Homo 56, 197-205.

Lele, S., McCulloch, C.E., 2002. Invariance, Identifiability and Morphometrics. J. Am. Stat. Assoc. 97, 796-806. 
Loth, S.R., İşcan, M.Y., 2000. Sex determination. In: Siegel, J.A., Saukko, P.J., Knupfer, G.C. (Eds.) Encyclopaedia of Forensic Sciences. Academic, San Diego, California, pp. 252260.

Loy, A., Boglione, C., Cataudella, S., 1999. Geometric morphometrics and morpho-anatomy: a combined tool in the study of sea bream (Sparus aurata, sparidae) shape. J. Appl. Icthyol. 15, 104-110.

O'Higgins, P., Chadfield, P., Jones, N., 2001. Facial growth and the ontogeny of morphological variation within and between the primates Cebus apella and Cercocebus torquatus. J. Zool. 254, 337-357.

Oettlé, A.C., Pretorius, E., Steyn, M., 2005. Geometric morphometric analysis of mandibular ramus flexure. Am. J. Phys. Anthropol. 128, 623-629.

Oettlé, A.C., Pretorius, E., Steyn, M., 2009. Geometric morphometric analysis of the use of mandibular gonial eversion in sex determination. Homo 60, 29-43.

Perez, S.I., Bernal, V., Gonzalez, P.N., 2006. Differences between sliding semi-landmark methods in geometric morphometrics, with an application to human craniofacial and dental variation. J. Anat. 208, 769-784.

Prescher, A., 2000. Anatomical basics, variations, and degenerative changes of the shoulder joint and shoulder girdle. Eur. J. Radiol. 35, 88-102.

Pretorius, E., Scholtz, C.H., 2001. Geometric morphometrics and the analysis of higher taxa: a case study based on the metendosternite of the Scarabaeoidea (Coleoptera). Biol. J. Linn. Soc. $74,35-50$.

Pretorius, E., Steyn, M., Scholtz, Y., 2006. An investigation into the usability of geometric morphometric analysis in assessment of sexual dimorphism. Am. J. Phys. Anthropol. 129, $64-70$.

Rohlf, F.J., 2000. Statistical power comparisons among alternative morphometric methods. Am. J. Phys. Anthropol. 111, 463-478.

Rohlf, F.J., 2003. Bias and error in estimates of mean shape in geometric morphometrics. J. Hum. Evol. 44, 665-683.

Scheuer, L., Black, S.M., 2000. Developmental juvenile osteology. Academic, San Diego (Calif).

Sheets, D.H., 2001. IMP, Integrated Morphometrics Package. Available from: URL: http://www.canisius.edu/ sheets/morphsoft.html; Sheets, David H. 
Slice, D.E., Bookstein, F.L., Marcus, L.F., Rohlf, F.J., 1998. A Glossary for Geometric Morphometrics (Part 2). Accessed 8 April 2010. Available from:

http://life.bio.sunysb.edu/morph/glossary/gloss2.html.

Slice, D.E., Bookstein, F.L., Marcus, L.F., Rohlf, F.J., 2009. A Glossary for Geometric Morphometrics (Part 1). Accessed 8 April 2010. Available from: http://life.bio.sunysb.edu/morph/glossary/gloss1.html.

Steyn, M., Pretorius, E., Hutten, L., 2004. Geometric morphometric analysis of the greater sciatic notch in South Africans. Homo 54, 197-206.

Strand Viðarsdóttir, U., O’Higgins, P., Stringer, C., 2002. A geometric morphometric study of regional differences in the ontogeny of the modern human facial skeleton. J. Anat. 201, 221-229.

Wolffson, D.M., 1950. Scapula shape and muscle function, with special reference to the vertebral border. Am. J. Phys. Anthropol. 8, 331-341. 


\section{Legends to Figures}

Fig. 1. Dorsal view of left scapula of an adult female to illustrate landmarks described in the text

Fig. 2. Consensus thin-plate splines for female and male scapulae: (A) female scapula and (B) male scapula

Fig. 3. Vector plot to show the separation of males (arrow points) from females (circles)

Fig. 4. Mean of the CVA plot for females and males $(-=$ females; $\mathbf{x}=$ males $)$. The large circle and cross represent the mean shape for females and males respectively

Fig. 5. Consensus thin-plate splines of the supraspinous fossa of female and male scapulae:

(A) supraspinous fossa of female scapula and (B) supraspinous fossa of male scapula

Fig. 6. Vector plot to show the separation of males (arrow points) from females (circles) along the supraspinous border

Fig. 7. Mean of the CVA plot for the supraspinous fossa border of females and males $(\mathbf{O}=$ females; $\mathbf{x}=$ males). The large circle and cross represent the mean shape for females and males respectively

Fig. 8. Consensus thin-plate splines for the curve data of females and males: (A) Curve data of females and (B) curve data for males

Fig. 9. Vector plot to show the separation of the male curve (arrows points) from the female curve (circles)

Fig. 10. Example of a typical female scapula.

Fig. 11. Example of a typical male scapula. 
Table 1. Percentage of males and females correctly assigned using a canonical variates analysis with the traditional landmark data. The number of individuals used is indicated in the left hand column

\begin{tabular}{|c|c|c|c|}
\hline Sex as in & \multicolumn{2}{|c|}{ CVA assignment based on shape data } & \multirow{4}{*}{$\begin{array}{l}\text { Percentage } \\
\text { correctly } \\
\text { assigned }\end{array}$} \\
\hline \multirow[t]{3}{*}{ dataset } & & & \\
\hline & Correctly & Incorrectly & \\
\hline & assigned & assigned & \\
\hline Females (45) & 41 & 4 & $91.11 \%$ \\
\hline Males (45) & 43 & 2 & $95.56 \%$ \\
\hline
\end{tabular}


Table 2. Percentage of males and females correctly assigned using a canonical variates analysis with the curve landmark data. The number of individuals used is indicated in the left hand column

\begin{tabular}{llll}
\hline Sex as in & CVA assignment based on shape data & $\begin{array}{l}\text { Percentage } \\
\text { correctly } \\
\text { Dataset }\end{array}$ & \\
& & & $\begin{array}{l}\text { assigned } \\
\end{array}$ \\
\cline { 2 - 3 } & Correctly & Incorrectly & \\
& assigned & assigned & \\
\hline Females (45) & 29 & 16 & $64.44 \%$ \\
Males (45) & 29 & 16 & $64.44 \%$ \\
\hline
\end{tabular}




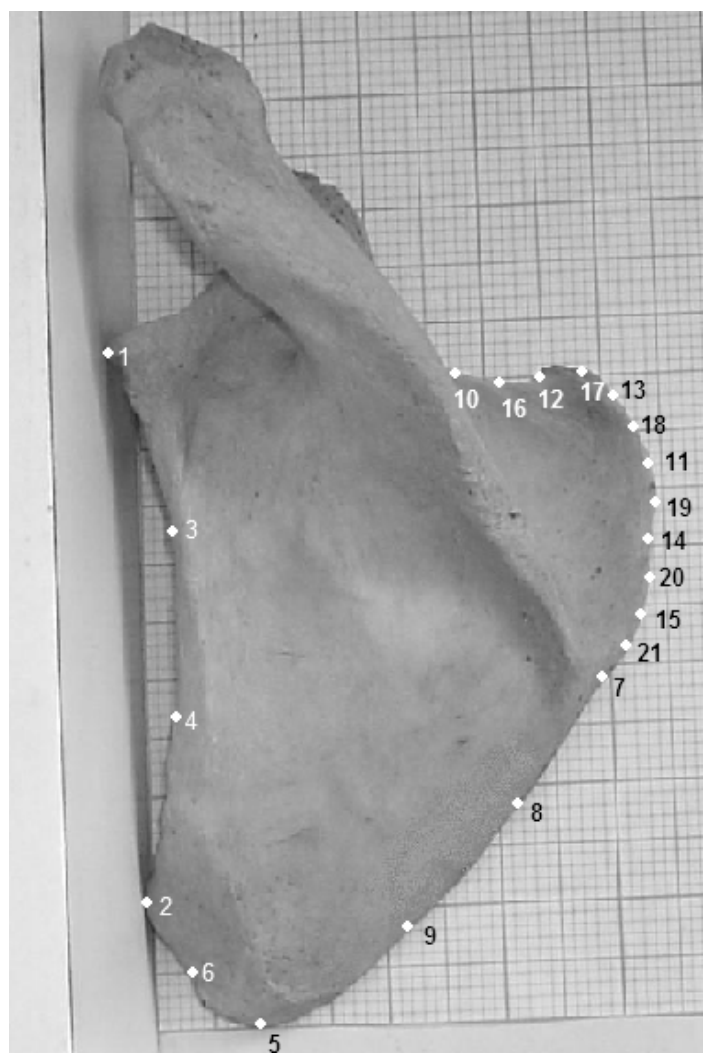

Fig. 1. Dorsal view of left scapula of an adult female to illustrate landmarks described in the text. 
A

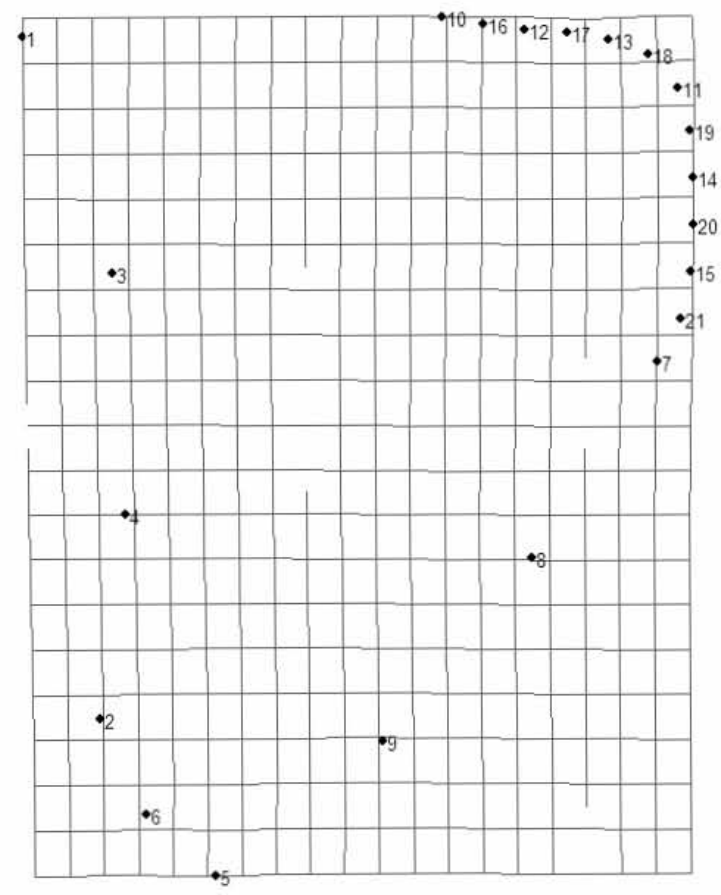

B

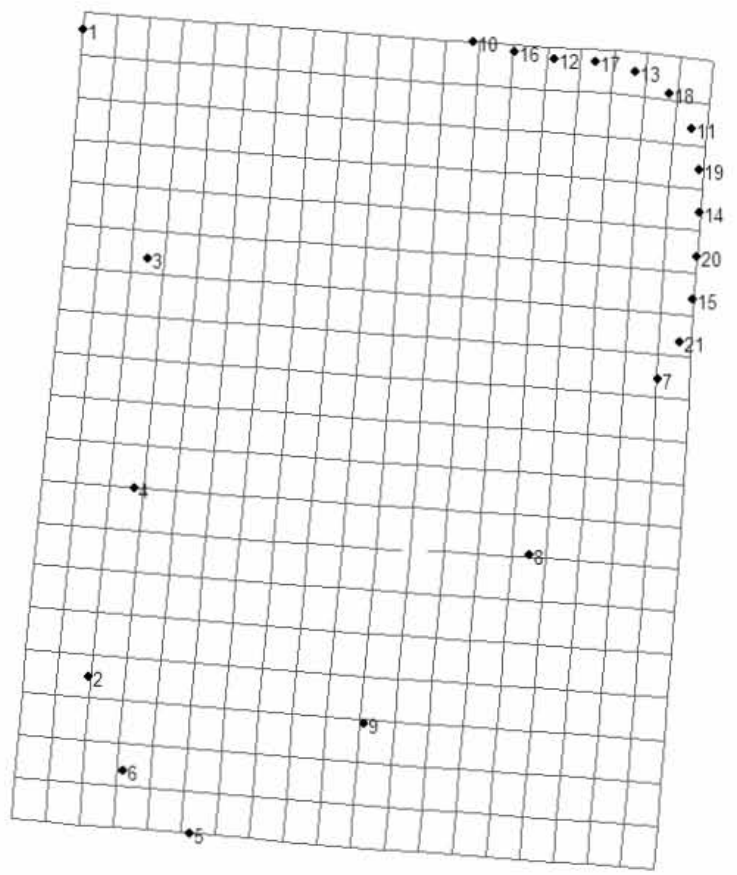

Fig. 2. Consensus thin-plate splines for female and male scapulae: (A) female scapula and (B) male scapula 

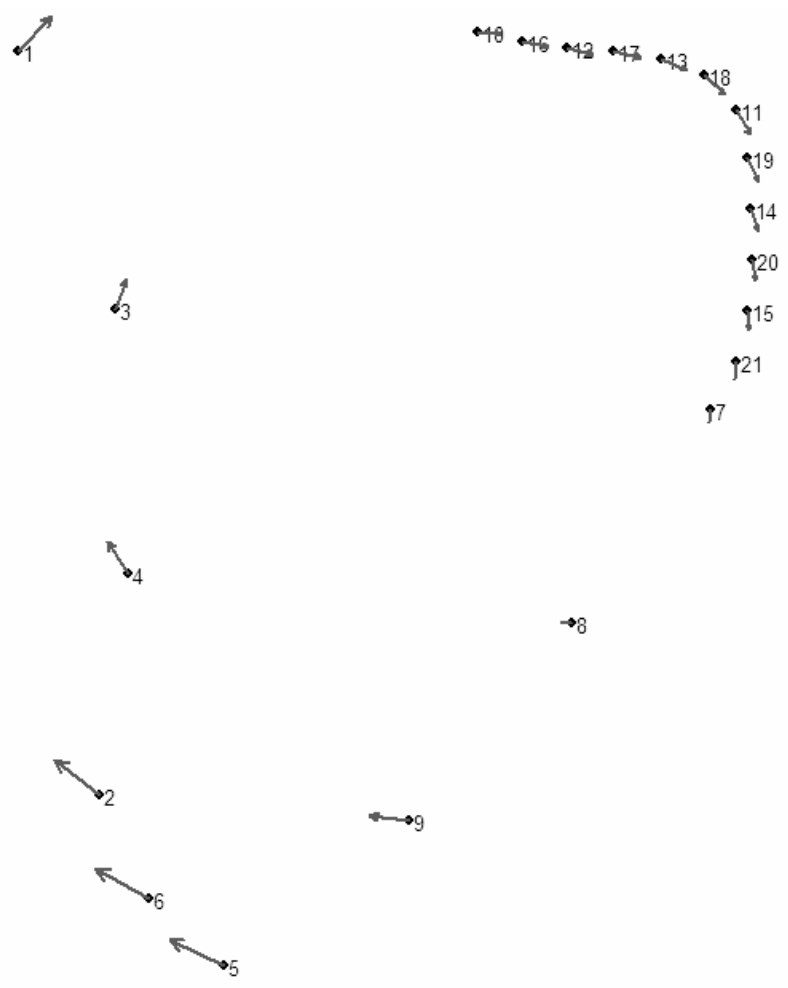

Fig. 3. Vector plot to show the separation of males (arrow points) from females (circles) 


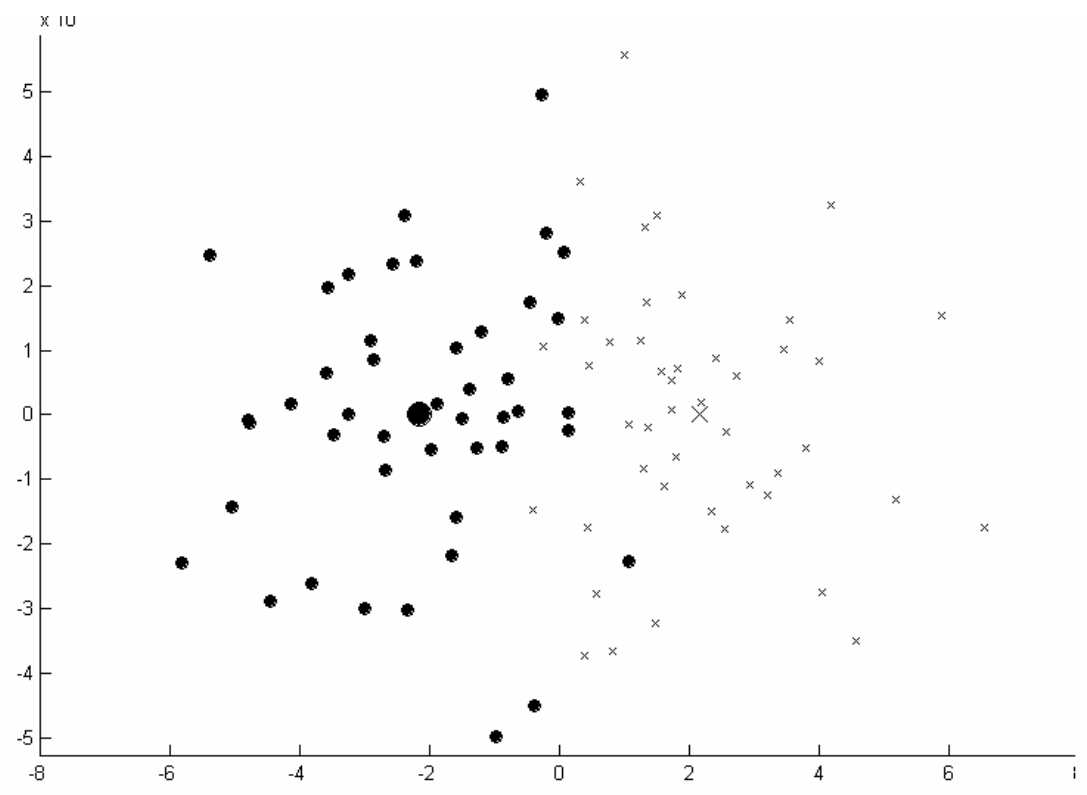

Fig. 4. Mean of the CVA plot for females and males ( $=$ females; $\mathbf{x}=$ males $)$. The larger circle and cross represent the mean shape for females and males respectively 
A

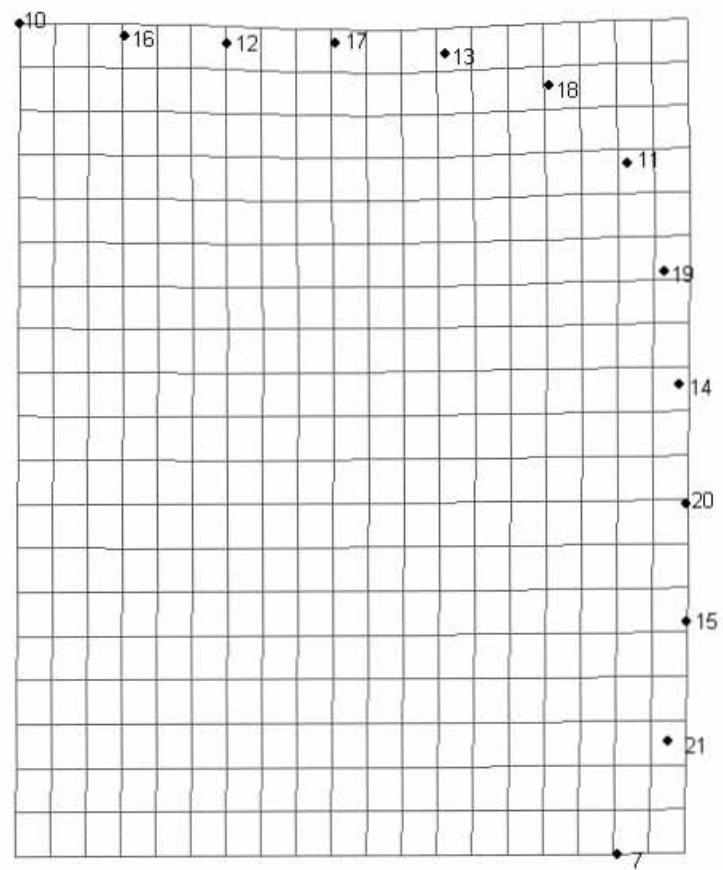

B

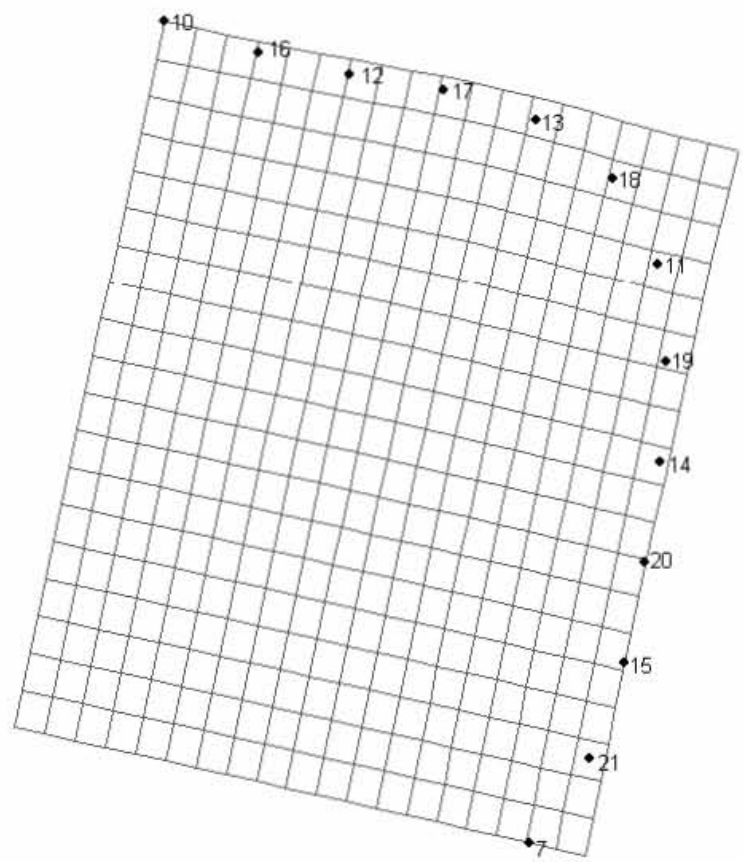

Fig. 5. Consensus thin-plate splines of the supraspinous fossa of female and male scapulae: (A) supraspinous fossa of female scapula and (B) supraspinous fossa of male scapula 


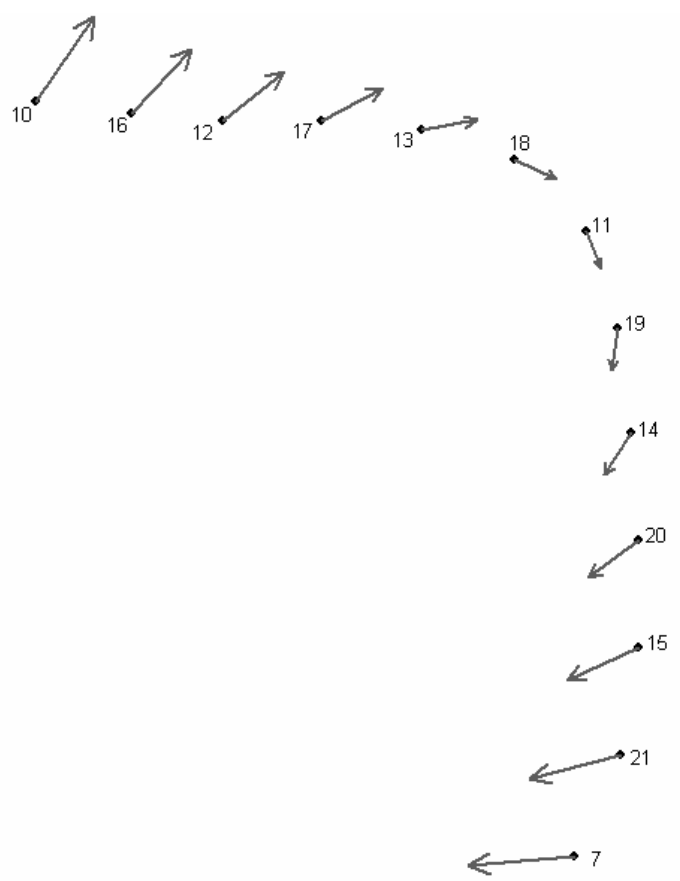

Fig. 6. Vector plot to show the separation of males (arrow points) from females (circles) along the supraspinous border

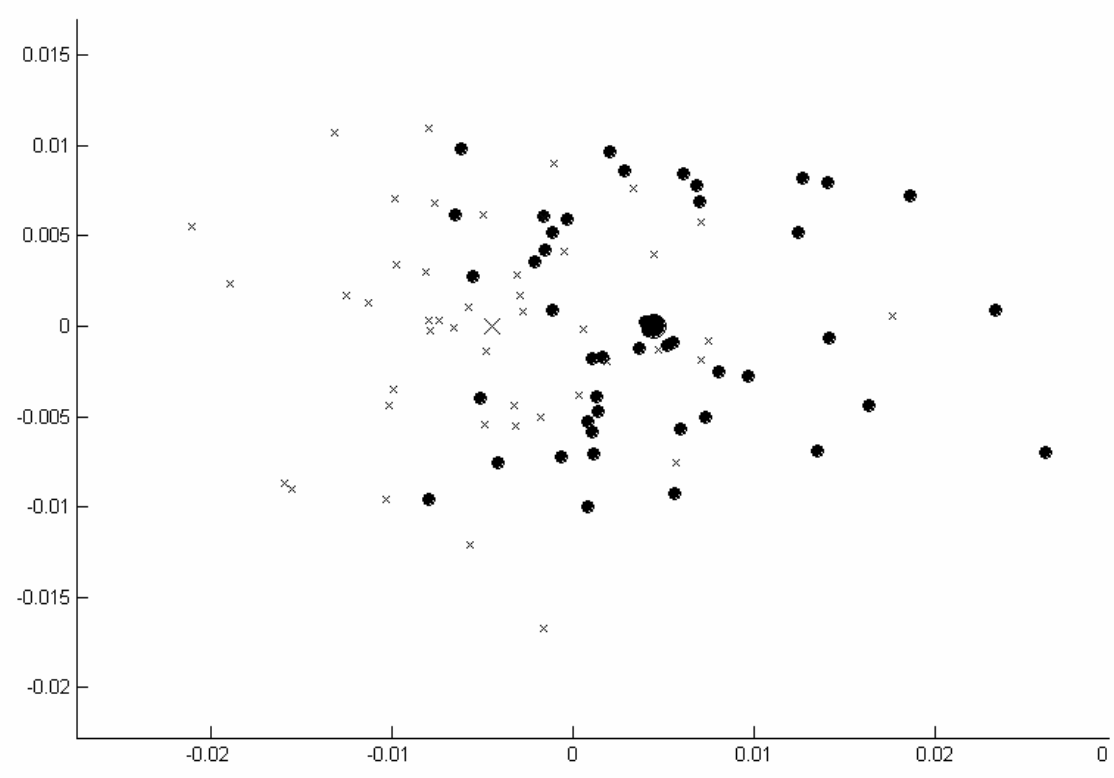

Fig. 7. Mean of the CVA plot for the supraspinous fossa border of females and males $(0=$ females; $\mathbf{x}=$ males). The larger circle and cross represent the mean shape for females and males respectively 
A

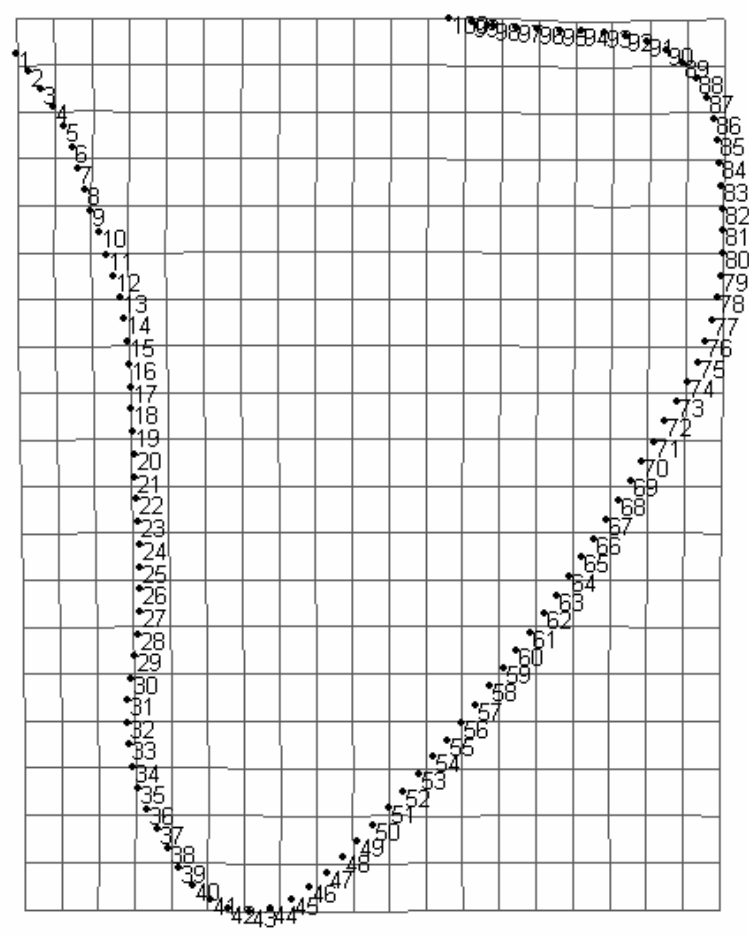

B

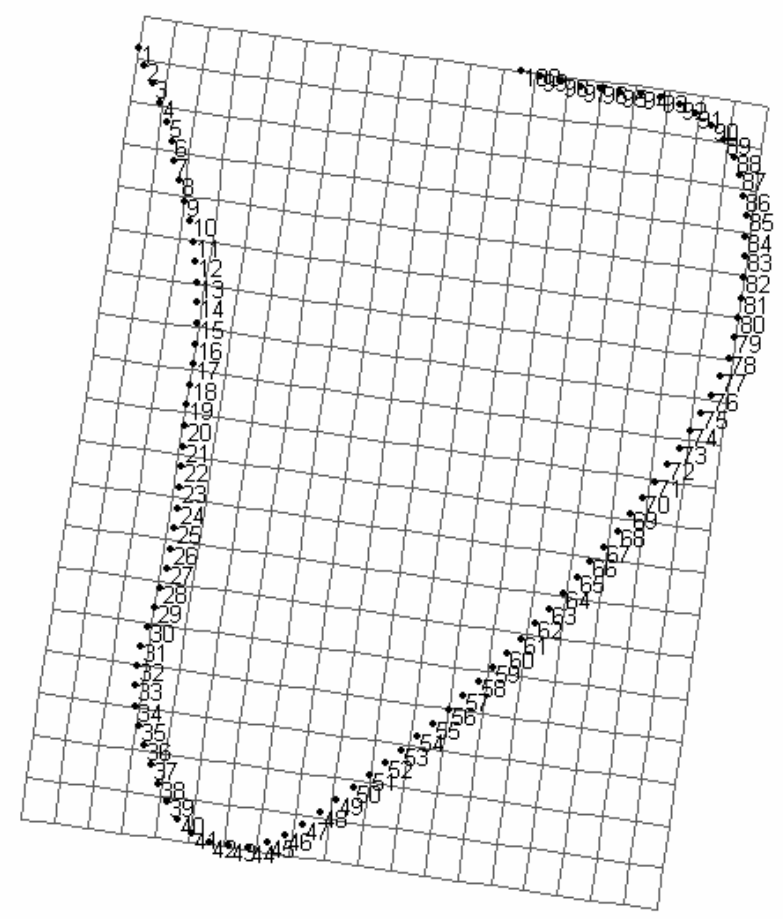

Fig. 8. Consensus thin-plate splines for the curve data of females and males: (A) Curve data of females and (B) curve data for males 


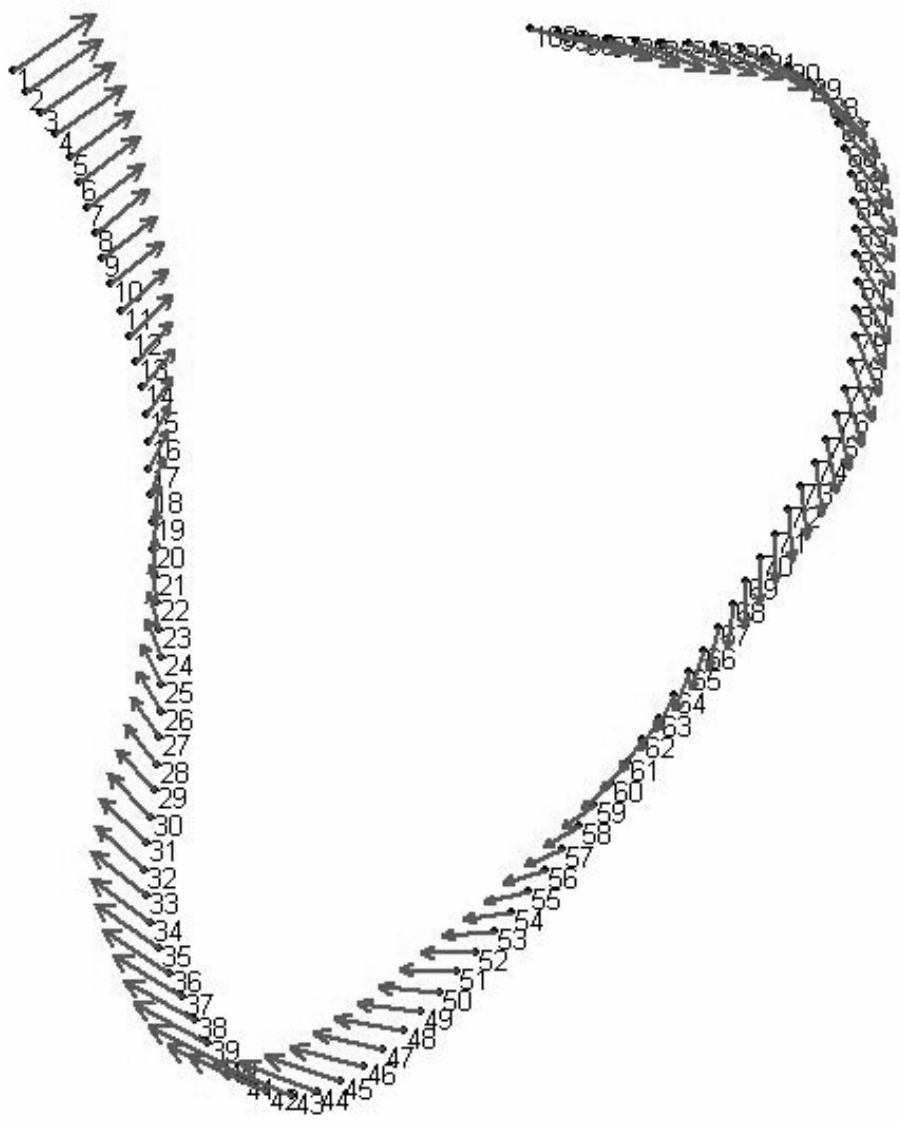

Fig. 9. Vector plot to show the separation of the male curve (arrows points) from the female curve (circles) 


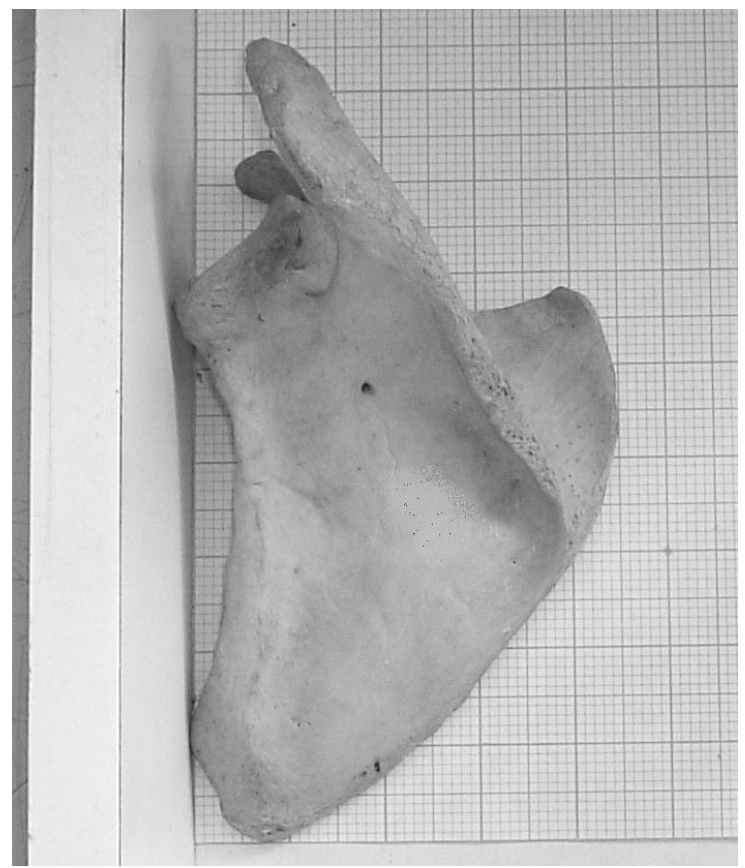

Fig. 10. Example of a typical female scapula. Note the narrow scapular body, the blunt inferior angle and straight medial border. Also note the convex superior border of the supraspinous fossa, as well as the straight medial border of the supraspinous fossa 


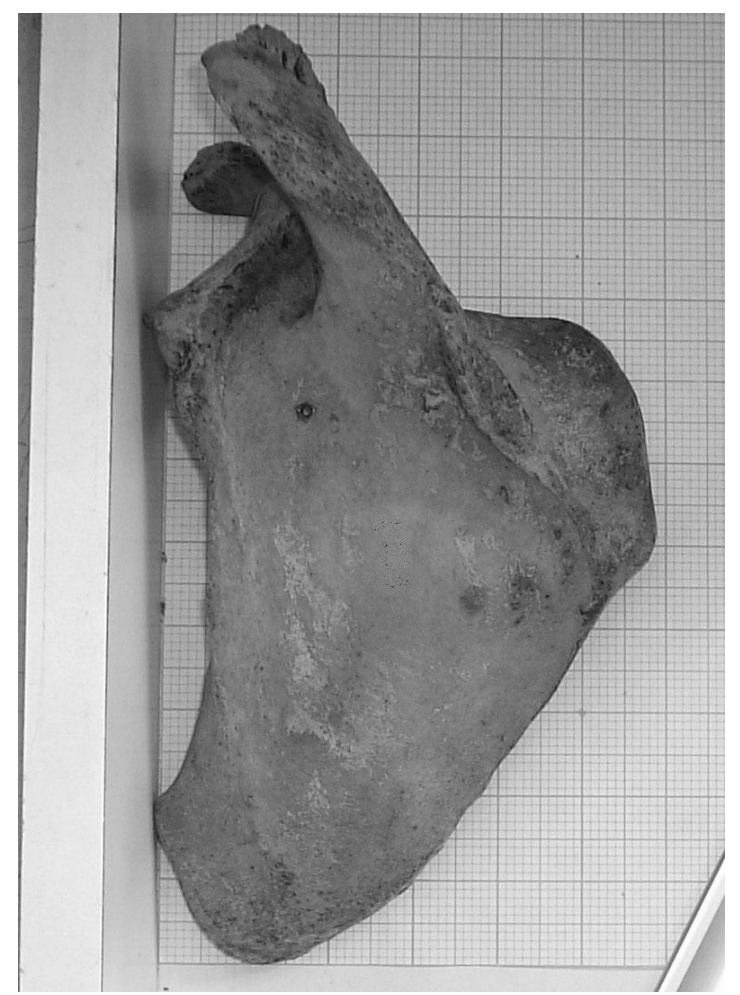

Fig. 11. Example of a typical male scapula. Note the scapular body which is broader than that of the female and the lateral border which is more curved. The medial border is also more curved than that of the female. The superior border of the supraspinous fossa is straighter than that of the female 